\title{
Association between Acute Coronary Syndrome and Helicobacter Pylori Chronic Infection? What data mining tells us?
}

\author{
Hossam Mansour ${ }^{1}$, Ramadan Ghaleb ${ }^{2 \dagger}$, Mohamed Fakhry ${ }^{3}$, Elsayed Elgohary ${ }^{4}$, Ali Ismael ${ }^{4}$, Abd- \\ Elrazek Abd-Elrazek ${ }^{*}$ \\ ${ }^{1}$ Department of Cardiology, 6th October University, Egypt \\ ${ }^{2}$ Department of Cardiology, Aswan University, Egypt \\ ${ }^{3}$ Department of Tropical Medicine, Al-Azhar University, Asuit Branch, Egypt \\ ${ }^{4}$ Department of Internal Medicine, Zagazig University, Egypt \\ ${ }^{5}$ Department of Tropical Medicine, Aswan University, Egypt \\ t; Equally Contributing First Author
}

Received: 23 March, 2017 ; Accepted: 12 April, 2017; Published: 21 April, 2017

*Corresponding author: Abd Elrazek M Ali Hussein MD. PHD, Liver Transplant, Department of Tropical and GIT diseases, Researcher; data mining in medicine, Aswan Faculty of Medicine, Aswan University; Aswan, PO 81528, Egypt, Tel: +201116414192, Fax: (+2)0973480134; E-mail: ahmadrazek@gmail.com

\section{Abstract}

Background: Acute Coronary Syndrome (ACS) is one of life threating conditions affecting all populations, even those without related risk factors. Meanwhile some studies shed light on chronic infections such as H.Pylori, Chlamydia, Epstein Bar virus and other micro-organisms may play a role initiating or and overexposing ACS, still not explaining the overall dilemma.

Aim: We aimed to evaluate any role association between $\mathrm{H}$. Pylori chronic infection and ACS using artificial intelligence programs.

Patients and Methods: Between August 2016 and March 2017, 125 patients presented with ACS were evaluated for Pylori ELIZA tests to discover any association using high performance computing analysis of Rapid I Software analysis.

Conclusion: Data mining analyses approved such an association between long duration intermittent chest pain related-ACS and Chronic Pylori positive IgG test, however many studied are ongoing for more explanation and much understanding.

Keywords: ACS; H. Pylori; Data mining

\section{Abbreviations}

ACS: Acute Coronary Syndrome; CHD: Chronic Heart disease; CVD: Cardiovascular diseases; DM: Data mining; H.Pylori: Helicobacter Pylori; PCI: Percutaneous Coronary Intervention

\section{Introduction}

Risk factors such as hypocholesteremia, hypertriglyceridemia, LDL and HDL, smoking, hypertension, family history or even co-morbidities like sedentary life, social stress and obesity do not explain ACS in many circumstances. Helicobacter pylori (H. pylori) is a Gram-negative spiral bacterium colonizing gastric mucosa of nearly half of human population was discovered by Marshall and Warren and for their breakthrough discovery associated peptic ulcerations induced by H.pylori, awarded Nobel Prize in 1999 [1,2]. A characteristic feature of H. pylori infection is an excessive inflammatory response may affect gastrointestinal tract or even extra-gastric organs. However the majority of H. pylori infections remain asymptomatic. Currently extra-gastric H.Pylori manifestations are well known such as sideroplastic anemia and vitamin B12 deficiency. Nevertheless cardiac diseases and atherosclerosis have been also reported $[3,4]$. Data mining programs being a break through resolution of bioinformatics related- big data analyses or even for prediction future medicine, may discover such dilemma of association, dissociation or partially associated organ- microorganism conditional disorders.

\section{Artificial Intelligence (AI) Analyses}

There are many Software intelligent programs related-big data analyses, one of those well-known- programs is Rapid I, Berlin, Germany with its rapidly consequent versions being one of the most popular computational analysis using with great success worldwide.

In current study we used both Naïve Bayes (10-cross validation method) and decision tree of stumping computational engineering application.

\section{Patients and Methods}

Prospectively for 125 patients; 95 Male; (74\%) and 30 Female (24\%), presented with typical chest pain. ECG and or PCI; Percutaneous Coronary Intervention revealed ACS have been investigated to H.Pylori infection by Ab test of both acute and chronic infection ; IgM and IgG tests respectively to evaluate the association between ACS and H.pylori infection

\section{Results}

According to data mining analysis of 125 patients presented with typical chest pain, we have to consider H.Pylori chronic infection as a 
risk factor for ACS of patients presented with long duration of intermittent chest pain; if H.Pylori IgG is being equal or more than 1.4 units it is highly indicated for ACS due to chronic H.Pylori infection (Possible independent factor). However if H.Pylori is being Positive but less than 1.4 units another factor (Age) should be considered (Diagram 1 of chart wise; decision tree).

IgG appear to be superior than IgM inducing ACS

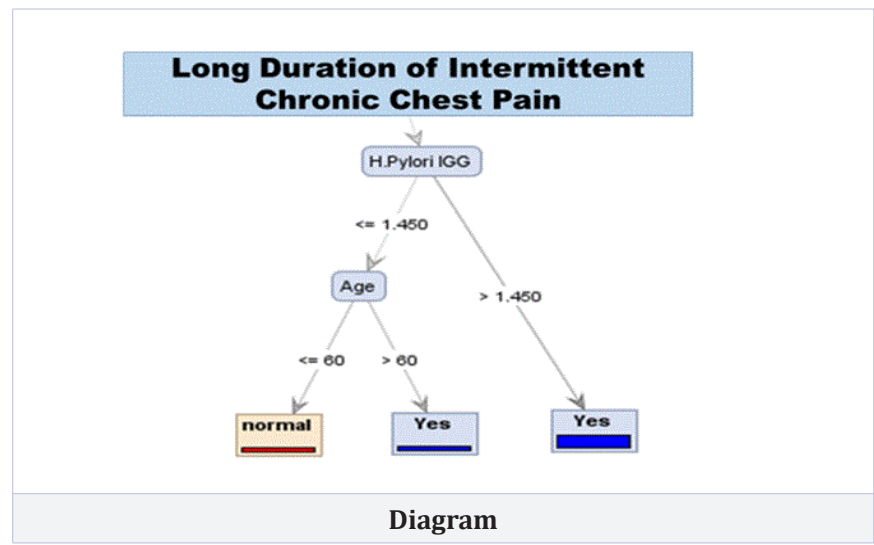

\section{Discussion}

H.Pylori is a Gram Negative, microaerophilic bacterium found mainly in the stomach, and may be present in other parts of the body, (Extra-Gastric H.Pylori). Recently, there is a documented association between chronic H.pylorichrnoic infections and extra gastric disorders including cardiovascular (CVD) diseases, has been recently investigated [5]. Since risk factors related-CVS do not explain all cases of coronary heart disease (CHD) the concept that atherogenesis may have infectious adjuvant role, recently helicobacter cinaedi may play a role in a proatherogenic antigen should be considered. The role of virus and bacterial pathogens including Helicobacter pylori (H. pylori), Epstein bar virus and other microbiotas are now considered as important co-adjuvant factors may implicated in the development of CHD. However it is still not clear if such chronic infections may influence the course of CHD via different mechanisms suchas direct chronic inflammatory reactions, cross antigenicity of immune complex processes or through modification of classic CHD risk factors such as hyperlipidemia [6]. The interesting finding of Mendall and colleagues published in 1994 showed that CHD patients have elevated levels of serum anti-H. Pylori antibodies [7] According to these pioneer findings, some authors confirm and some exclude the existence of this connection making the matter in a debate discussion. Moreover still there is no consensus proofs on the role of $\mathrm{H}$. pylori in either initiation or progression of CHD. In order to describe the involvement of $\mathrm{H}$. pylori in the development of CHD, it is necessary to find the largest number of reliable research studies; big data confirming this relationship. For our knowledge there is no study have used data mining analysis of such big data related ACS and Pylori, that artificial intelligence of Rapid I used in the current study can find the accurate or possible situation associations, hence according to our results only ACS of long duration of intermittent typical chest pain may becaused by Pylori chronic infection [8-20]. Data mining is the breakthrough in economy, biology, trading, business, astronomy and medicine [21-23]. Data mining can predict disease related-mortality and morbidity that will influence the overall medical progress [24-26]. In current research decision tree of Rapid I software program can detect the cut off point for ACS related - chronic Pylori infection at 1.4IU of IgG test, hence there was a good correlation between long duration of chronic chest pain and ACS due to Pylori, nevertheless values less than 1.4IU but still positive to IgG should be evaluated using age as a second predictor factor for ACS (Figure 1).

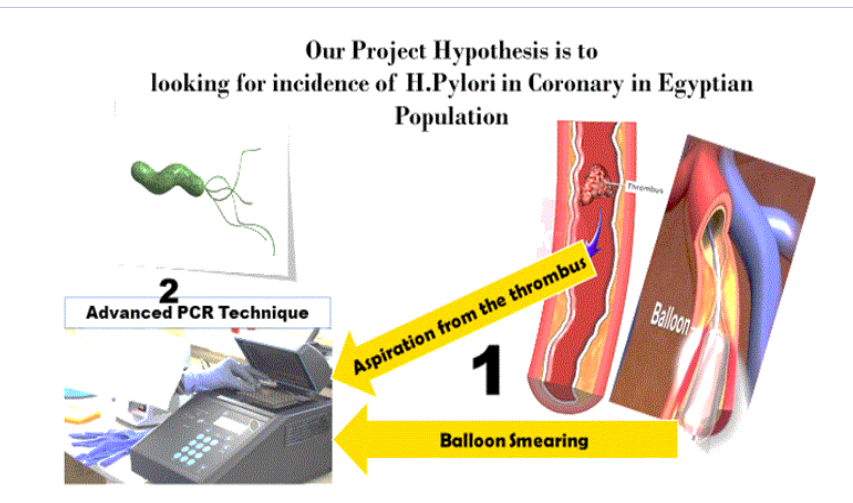

Figure 1: showing a brief explanation of our dedicated ongoing project study

Nevertheless Pylori should be treated not only for the therapy of gastrointestinal diseases and for prevention of gastric lymphoma only, but also because Pylori may be considered a risk factor for ACS especially in those without fully explained risk factors. However many studies should be planned to estimate the real role of Pylori and other opportunistic or chronic infection in developing ACS.

\section{Limitation of the study}

1. Hence data mining mainly deals with big data analyses, but preliminary results may be helpful in ongoing research studies.

\section{All patients were Egyptians.}

\section{Future Recommendation}

There is a multi-center big project study to detect H.Pylori and other micro-organisms in atheromatous coronary plaque (Ongoing Egyptian Multi-center Project; Aswan university, Zewail City for Biomedicine and Technology, Zagazig University, 6th October university , other university hospitals, Ministry of health hospitals and private hospitals)

\section{References}

1. Chmiela M, Kowalewicz-Kulbat M, Miszczak A, Wisniewska M, Rechcinski T, Kolodziej K, et al. A link between Helicobacter pylori and/ or Chlamydia spp. Infections and atherosclerosis. FEMS Immunol Med Microbiol 2003;36(3):187-192.

2. Park MJ, Choi SH, Kim D, Kang SJ, Chung SJ, Choi SY, et al. Association between Helicobacter pylori Seropositivity and the Coronary Artery Calcium Score in a Screening Population. Gut Liver. 2011;5(3):321-327. doi: $10.5009 / \mathrm{gnl} .2011 .5 .3 .321$

3. Vafaeimanesh J, Hejazi SF, Damanpak V, Vahedian M, Sattari M, Seyyedmajidi M. Association of Helicobacter pylori infection with coronary artery disease: is Helicobacter pylori a risk factor? ScientificWorldJournal. 2014;2014:516354. doi: 10.1155/2014/516354

4. Pasceri V, Cammarota G, Patti G, Cuoco L, Gasbarrini A, Grillo RL, et al. Association of virulent Helicobacter pylori strains with ischemic heart disease. Circulation. 1998;97(17):1675-1679

5. Chmiela M, Gajewski A, Rudnicka K. Helicobacter pylori vs coronary heart disease - searching for connections. World J Cardiol. 2015;7(4):187203. doi: 10.4330/wjc.v7.i4.187.

6. M. de Bernard, et al. HC-NAP is a pro-atherogenic antigen produced by Hylicobactercinaedi. European Helicobacter and Microbiota Study Group Conference, Magdeburg, Germany, September 2016. 
7. Mendall MA, Goggin PM, Molineaux N, Levy J, Toosy T, Strachan D, et al. Relation of Helicobacter pylori infection and coronary heart disease. $\mathrm{Br}$ Heart J. 1994;71(5):437-439

8. Khedmat H, Karbasi-Afshar R, Agah S, Taheri S. Helicobacter pylori Infection in the general population: A Middle Eastern perspective. Caspian J Intern Med. 2013;4(4):745-753

9. Hassanin N, Gharib S, El Ramly MZ, Meged MA, Makram A. Metabolic syndrome and coronary artery disease in young Egyptians presenting with acute coronary syndrome. Kasr Al Ainy Med J. 2015;21(1):27-33. doi: $10.4103 / 1687-4625.155666$

10. Sun J, Rangan P, Bhat SS, Liu L. A meta-analysis of the association between Helicobacter pylori infection and risk of coronary heart disease from published prospective studies. Helicobacter. 2016;21(1):11-23. doi: 10.1111/hel.12234

11.Said Mohammad Zade M, Eishi A, Behrozian R, Rahimi E. Relationship between Helicobacter pylori infection and cardiac syndrome X. J Shahrekord University Med Sci. 2009;11(1):58-63.

12.Eskandarian R, Malek M, Mousavi SH, Babaei M. Association of Helicobacter pylori infection with cardiac syndrome X. Singapore Med J. 2006;47(8):704-706

13. Sung KC, Rhee EJ, Ryu SH, Beck SH. Prevalence of Helicobacter pylori infection and its association with cardiovascular risk factors in Korean adults. Int J Cardiol. 2005;102(3):411-417.

14. Ahmed N, Tenguria S, Nandanwar N. Helicobacter pylori--a seasonedpathogen by any other name. Gut Pathog. 2009;1:24. doi: 10.1186/1757-4749-1-24

15.Leja M, Cine E, Rudzite D, Vilkoite I, Huttunen T, Daugule I, et al. Prevalence of Helicobacter pylori infection and atrophic gastritis in Latvia. Eur J Gastroenterol Hepatol. 2012;24(12):1410-1417. doi: 10.1097/ MEG.0b013e3283583ca5

16. Sasazuki S, Inoue M, Iwasaki M, Otani T, Yamamoto S, Ikeda S et al. Effect of Helicobacter pylori infection combined with caga and pepsinogen status on gastric cancer development among Japanese men and women: A nested case-control study. Cancer Epidemiol Biomarkers Prev. 2006;15(7):13411347

17.Ferreccio C, Rollan A, Harris PR, Serrano C, Gederlini A, Margozzini P et al. Gastric cancer is related to early Helicobacter pylori infection in a highprevalence country. Cancer Epidemiol Biomarkers Prev. 2007;16(4):662667

18. Wang X, Terry P, Yan H. Stomach cancer in 67 Chinese counties: evidence of interaction between salt consumption and Helicobacter pylori infection. Asia Pac J ClinNutr. 2008; 17(4):644-650

19. Torres J, Leal-Herrera Y, Perez-Perez G, Gomez A, Camorlinga-Ponce M, Cedillo-Rivera R, et al. A community-based seroepidemiologic study of Helicobacter pylori infection in Mexico. J Infect Dis. 1998;178(4):10891094.

20.AbdElrazekAbdElrazek, Ahmed Elbanna. Validation of Data miningin clinical medicine. Appl. Math. Inf. Sci. 2016;10(4):1637-1640

21. AbdElrazek A, Amer M, El-Hawary B, Salah A, Bhagavathula AS, Alboraie $\mathrm{M}$, et al. Prediction of $\mathrm{HCV}$ vertical transmission: what are factors should be optimized using data mining computational analysis. Liv Int. 2016 .

22. AbdElrazek AE, Bilasy SE, Elbanna AE, Elsherif Asim. Prior to the Oral Therapy, What Do We Know About HCV-4 in Egypt: A RandomizedSurvey of Prevalence and Risks Using Data Mining Computed Analysis. Medicine (Baltimore). 2014;93(28):e204. doi: 10.1097/MD.0000000000000204

23. AbdElrazek M Ali, H Mahfouz. Prediction of esophageal variceal degrees using data mining: is validated in clinical medicine? Global J Comp Sci
Tech. 2013;13:10-C.

24. AbdElrazek AE, Mahfouz HM, Metwally AM, ElShamy AM. Mortality prediction of nonalcoholic patients presenting with upper gastrointestinal bleeding using data mining. Eur J Gastroenterol Hepatol 2014;26(2):187191

25. Lu S, Jin B, Cowart LA, Lu X. From Data towards Knowledge: Revealing the Architecture of Signaling Systems by Unifying Knowledge Mining and Data Mining of Systematic Perturbation Data. Plos One. 2013;8(4):e61134 